\title{
Optical properties of light-hole excitons in GaN epilayers
}

\author{
Fan Zhang, ${ }^{1}$ S. J. Xu, ${ }^{1, a)}$ J. Q. Ning, ${ }^{1}$ C. C. Zheng, ${ }^{1}$ D. G. Zhao, ${ }^{2}$ H. Yang, ${ }^{3}$ and \\ C. M. Che \\ ${ }^{1}$ Department of Physics and HKU-CAS Joint Laboratory on New Materials, The University of Hong Kong, \\ Pokfulam Road, Hong Kong, China \\ ${ }^{2}$ State Key Laboratory on Integrated Optoelectronics, Institute of Semiconductors, Chinese Academy \\ of Sciences, Beijing 100083, China \\ ${ }^{3}$ Suzhou Institute of Nano-tech and Nano-bionics, Chinese Academy of Sciences, Suzhou 215125, China \\ ${ }^{4}$ Institute of Molecular Functional Materials, HKU-CAS Joint Laboratory on New Materials, \\ and Department of Chemistry, The University of Hong Kong, Pokfulam Road, Hong Kong, China
}

(Received 8 September 2010; accepted 17 October 2010; published online 15 December 2010)

\begin{abstract}
Optical properties of light-hole free exciton $\left(\mathrm{FX}_{\mathrm{B}}\right)$ in $\mathrm{GaN}$ epilayers were investigated by using near-resonance photoluminescence (PL) and time-resolved PL techniques. In contrast to the case of off-resonance PL where only heavy-hole free excitons $\left(\mathrm{FX}_{\mathrm{A}}\right)$ have strong response, $\mathrm{FX}_{\mathrm{B}}$ band can be well resolved in the near-resonance PL spectra. The variable-temperature near-resonance PL spectra show that the linewidth of $\mathrm{FX}_{\mathrm{B}}$ broadens faster than the $\mathrm{FX}_{\mathrm{A}}$ with increasing temperature. Moreover, the luminescence lifetime of $\mathrm{FX}_{\mathrm{B}}$ is found to be shorter than that of $\mathrm{FX}_{\mathrm{A}}$. () 2010 American Institute of Physics. [doi:10.1063/1.3520218]
\end{abstract}

As an important semiconductor material used in shortwavelength optoelectronic devices as well as high-power and high-temperature electronic devices, ${ }^{1} \mathrm{GaN}$ and related materials have attracted tremendous interest. In the past years, massive research has been done on the fundamental understandings about the near-band-edge luminescence, ${ }^{2}$ temperature dependence of band gap and luminescence linewidth, ${ }^{3}$ impurities and dislocations in GaN, and so on. However, such a situation is still maintaining: Fundamental understanding has still been struggling to catch up rapid empirical development in this field. For example, little known is about light-hole free exciton $\mathrm{B}\left(\mathrm{FX}_{\mathrm{B}}\right)$ due to its fast decay to the heavy-hole band via nonradiative process. It is known that the heavy- and light-hole bands in wurtzite $\mathrm{GaN}$ are energetically spilt $(\sim 6 \mathrm{meV})$ at zone center. ${ }^{4}$ In the most PL spectra of $\mathrm{GaN}$ reported so far, the heavy-hole free exciton $\mathrm{A}\left(\mathrm{FX}_{\mathrm{A}}\right)$ emission line is dominant when $\mathrm{FX}_{\mathrm{B}}$ usually appears only as a weak shoulder which is hard to be spectrally resolved and investigated. On the other hand, the spectral structures associated with $\mathrm{FX}_{\mathrm{B}}$ can be well resolved in low temperature reflectance spectra of GaN with good quality ${ }^{5}$ due to the resonant excitation effect. Inspired by the resonance reflectance results, near-resonance PL was used to investigate the optical properties of $\mathrm{FX}_{\mathrm{B}}$ in $\mathrm{GaN}$ in this study. Some exciting results about the light-hole free excitons in $\mathrm{GaN}$ have been obtained.

The GaN epilayer used in this study was grown by metal-organic chemical vapor deposition on sapphire substrate. It was an intentionally undoped sample with the background carrier concentration of $5 \times 10^{15} \mathrm{~cm}^{-3}$. X-ray diffraction (XRD) measurements show that then the linewidths of diffraction peaks are 180 arcsec for both (002) and (102) planes. All these data indicate the high quality of the sample. In the variable-temperature near-resonance PL measure-

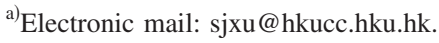

ments, the excitation light was achieved by dispersing a high pressure Xenon arc lamp with an Acton SP305 monochromator equipped with 1200 lines/mm grating. Its wavelength was set to be $354 \mathrm{~nm}$. The sample was mounted on the cold finger of a Janis closed-cycle cryostat with varying temperature range from 10 to $300 \mathrm{~K}$. The emission PL signals from the sample were dispersed by an Acton SP307 monochromator equipped with 1200 lines/mm grating and then were detected by a Hamamatsu R928 photomultiplier. Standard lock-in amplifier technique was used to maximum the signalto-noise ratio. Time-resolved PL was also employed to study the recombination dynamics of the free excitons in $\mathrm{GaN}$ in this study. A mode locked femtosecond (fs) Ti:sapphire laser (Tsunami) pumped by a $8 \mathrm{~W}$ solid state laser (Millennia) emitting at $532 \mathrm{~nm}$ was used as the pulse light source. This laser set produces $\sim 80$ fs pulses in the wavelength range $700-1000 \mathrm{~nm}$ at a repetition frequency of $82 \mathrm{MHz}$. Ultraviolet fs pulses were achieved by frequency doubling of the output of Ti:sapphire with a $\beta-\mathrm{BaB}_{2} \mathrm{O}_{4}(\mathrm{BBO})$ nonlinear optical crystal. The GaN sample was loaded in a $77 \mathrm{~K}$ Dewar and excited with pulsed laser at $502 \mathrm{~nm}$. The signal was collected by a Hamamatsu C4334 streak camera with a time resolution about 15 ps in conjunction with a Chromex 250i monochromator. In addition, the low-temperature reflectance spectra of the sample were also measured. The details of the experimental arrangement for measuring almost normal incident reflectance spectra have been described elsewhere. ${ }^{5}$

Figure 1 shows the near-resonance PL results at $15 \mathrm{~K}$ together with the off- resonance PL spectra (excited by a $\mathrm{He}-\mathrm{Cd} 325 \mathrm{~nm}$ laser) and reflectance spectra. Resolved and sharp peaks in the off-resonance PL spectrum indicate the high quality of the sample. $\mathrm{FX}_{\mathrm{B}}$ emission line is identified and well resolved in the near-resonance PL spectra. Therefore, near-resonance PL technique provides us a useful approach to investigate the optical properties of free exciton B in $\mathrm{GaN}$ epilayers. 


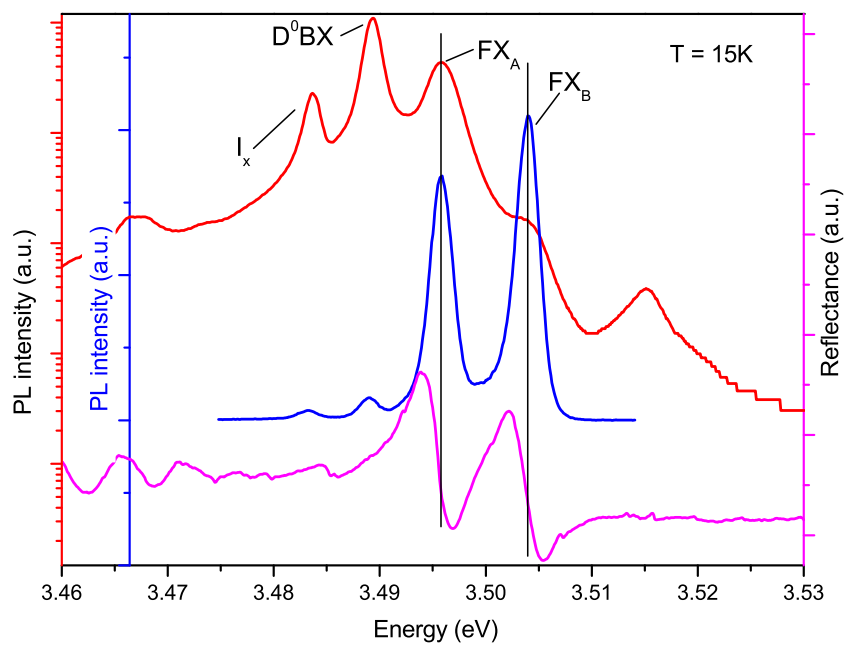

FIG. 1. (Color online) Off-resonance PL (semilog plot) spectrum (top), near-resonance PL spectrum (middle), and reflectance spectrum (bottom) of sample at $15 \mathrm{~K}$.

As shown in Fig. 2, the reflectance and near-resonance PL spectra can be simulated by using the interacting excitonic polariton damping model ${ }^{5-7}$ and Gaussian lineshape function, respectively. For the best simulation to the reflectance spectrum, adopted resonance energy of $\mathrm{A}$ and $\mathrm{B}$ excitonic polaritons was $3.4931 \pm 0.0005 \mathrm{eV}$ and $3.5005 \pm 0.0005 \mathrm{eV}$, respectively, and the polarizabilities of $\mathrm{A}$ and $\mathrm{B}$ excitonic polaritons were 0.018 and 0.012 , respectively. For the best fitting to the near-resonance PL spectrum, the energy of $\mathrm{FX}_{\mathrm{A}}$ and $\mathrm{FX}_{\mathrm{B}}$ lines was taken to be $3.4946 \pm 0.0002 \mathrm{eV}$ and $3.5027 \pm 0.0002 \mathrm{eV}$ at $10 \mathrm{~K}$, respectively, while their full width at half maximum (FWHM) was used as $2.80 \mathrm{meV}$ and $2.83 \mathrm{meV}$, respectively. The weak oscillatory structures in the reflectance spectra at lower energy side are mainly due to the thin-film interference effect.

Variable-temperature near-resonance PL measurements were also carried out on the sample for a temperature range from 8 to $120 \mathrm{~K}$. The results were shown in Fig. 3. The energy difference between $\mathrm{FX}_{\mathrm{A}}$ and $\mathrm{FX}_{\mathrm{B}}$ line is determined

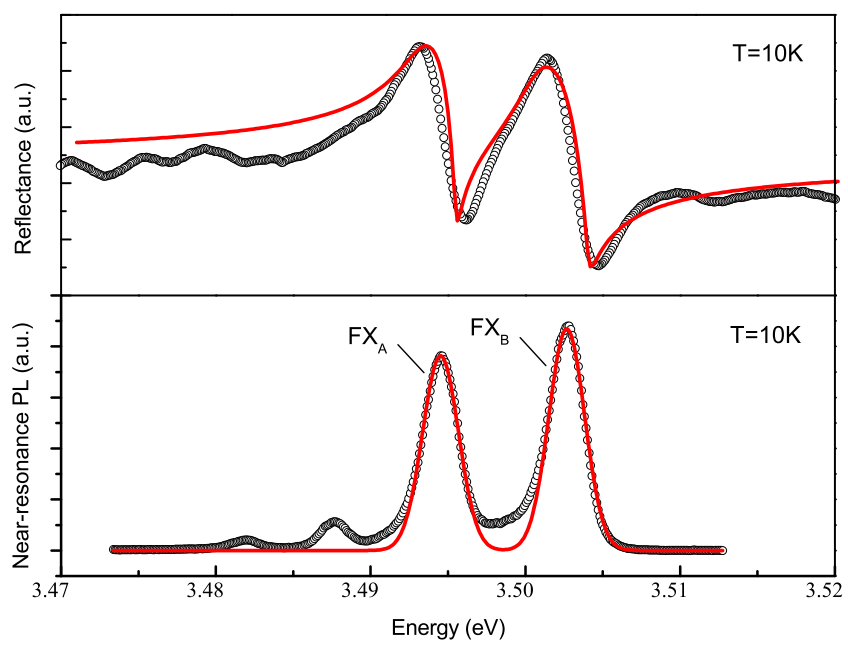

FIG. 2. (Color online) Low-temperature $(10 \mathrm{~K})$ reflectance and nearresonance PL spectra of GaN (circle symbols) and the fitting curves (solid lines).

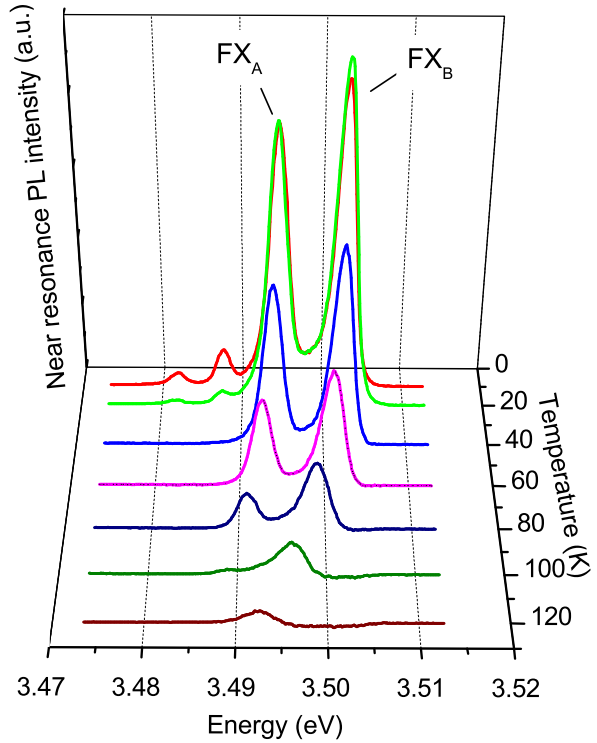

FIG. 3. (Color online) Variable-temperature near-resonance PL spectra of $\mathrm{GaN}$.

to be $8.1 \mathrm{meV}$ directly from the near-resonance PL spectra and keeps a constant within the measured temperature range. As expected, however, both $\mathrm{FX}_{\mathrm{A}}$ and $\mathrm{FX}_{\mathrm{B}}$ lines broaden with increasing temperature. Because the broadening effect is mainly due to the scattering by phonons in the sample, the temperature dependence of the free exciton linewidth can provide useful information on the exciton-phonon interactions. Figure 4 shows the temperature dependence of the FWHM of the $\mathrm{FX}_{\mathrm{A}}$ line (circles) and the $\mathrm{FX}_{\mathrm{B}}$ line (stars). It is found that the linewidth of $\mathrm{FX}_{\mathrm{B}}$ exhibits a faster broadening than that of $\mathrm{FX}_{\mathrm{A}}$ as the temperature increases. According to the reports by Rudin et al. ${ }^{8}$ and $\mathrm{Xu}$ et al.,${ }^{9}$ at low temperatures, exciton-acoustic phonon scattering is a dominate broadening mechanism and linewidth of free exciton emission lines shall exhibit a linear dependence on temperature, such as, $\Gamma(T) \approx \Gamma_{0}+\alpha T$. By using this linear dependence formula, the values of the parameter $\alpha_{\mathrm{A}}$ and $\alpha_{\mathrm{B}}$ are found to be $7.0 \mu \mathrm{eV} / \mathrm{K}$ and $16.3 \mu \mathrm{eV} / \mathrm{K}$ for $\mathrm{FX}_{\mathrm{A}}$ and $\mathrm{FX}_{\mathrm{B}}$, respec-

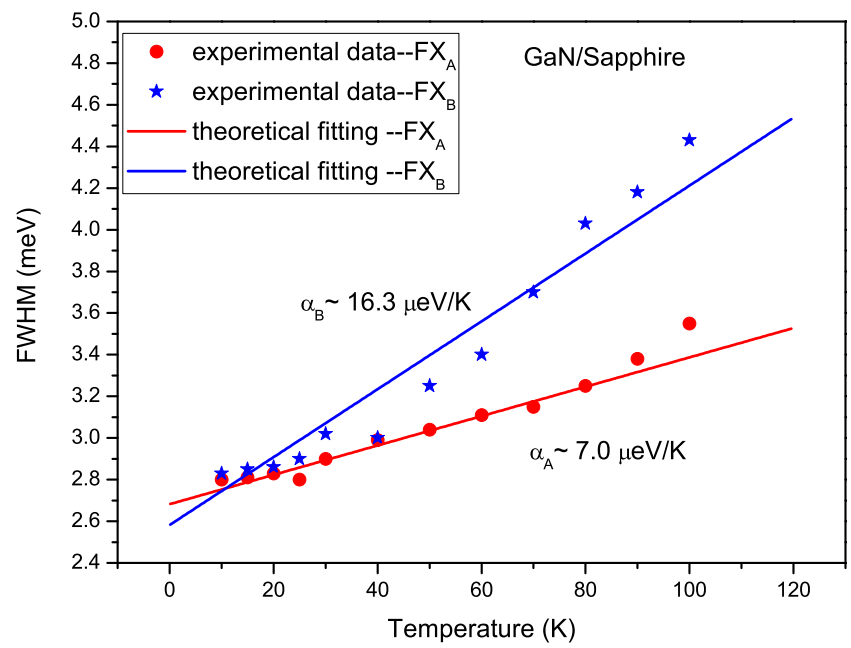

FIG. 4. (Color online) Temperature dependence of the linewidths of free exciton A and B in GaN. The symbols represent the experimental data while the solid lines stand for the fitting results. 


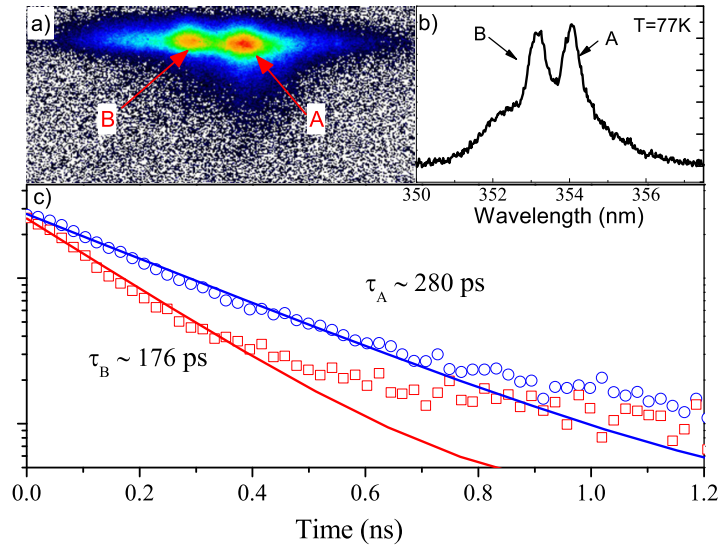

FIG. 5. (Color online) (a) A streak camera image of the band-edge luminescence of $\mathrm{GaN}$ at $77 \mathrm{~K}$; (b) A spectral profile of the streak camera image; (c) The intensity decay results of the two main emission lines (semilog plot) vs time. The circles represent the data for free exciton $\mathrm{A}$ and the squares for free exciton B. The solid lines are the fitting curves to the experimental data with a single exponential decay function.

tively. The fitting lines are plotted in Fig. 4. These results indicate that the light-hole free excitons experience a stronger acoustic phonon scattering than the heavy-hole free excitons in $\mathrm{GaN}$. A reasonable explanation is that the light-hole free excitons have a larger kinetic energy and a smaller effective mass than the heavy-hole free excitons.

In order to get more information of $\mathrm{FX}_{\mathrm{A}}$ and $\mathrm{FX}_{\mathrm{B}}$, timeresolved PL measurements were performed on the sample at $77 \mathrm{~K}$. Figure 5(a) shows a streak camera image of the bandedge luminescence of the GaN sample under the nearresonance excitation conditions. As expected, $\mathrm{FX}_{\mathrm{A}}$ and $\mathrm{FX}_{\mathrm{B}}$ emission lines can be well resolved in the image, as shown in Fig. 5(b). In Fig. 5(c), the intensity decaying curves (symbols) of these two lines versus time are plotted and can be fitted well by using a single exponential decay function (solid lines) except in the long decay time region where the luminescence signal becomes very weak and noisy. The fluorescence lifetime of $\mathrm{FX}_{\mathrm{A}}$ and $\mathrm{FX}_{\mathrm{B}}$ are found to be $\sim 280$ ps and $\sim 176 \mathrm{ps}$, respectively. According to a theoretical analysis by Toyozawa, ${ }^{10}$ the radiative lifetime of free excitons can be expressed as follows:

$$
\tau_{r A(B)}=\frac{1}{2}\left(\frac{5 \pi}{3}\right)^{3 / 5}\left(\frac{\hbar^{2} c^{7} M^{2} \beta_{A(B)} D^{3}}{v_{0}^{2} C^{4} \omega_{A(B)}}\right)^{1 / 5} \frac{1}{\omega_{A(B)}},
$$

in which $\beta_{A(B)}$ is the polarizability of free exciton $A(B)$, $M / v_{0}$ is the mass per unit volume of the unit cell, $D$ is the thickness of the sample film, $C$ is the deformation potential, and $\hbar \omega_{A(B)}$ is the energy of free exciton $A(B)$. This theoretical model predicts that light-hole free excitons shall have a shorter radiative time than heavy-hole free excitions. Clearly, our time-resolved PL data are consistent with the prediction of Toyozawa model.

In conclusion, the optical properties of free exciton B in GaN epilayers have been investigated by using the nearresonance PL and time-resolved PL techniques. The emission line from free exciton B is clearly resolved in the lowtemperature near-resonance PL spectra. The variabletemperature near-resonance PL spectra show that the linewidth of $\mathrm{FX}_{\mathrm{B}}$ broadens faster than that of $\mathrm{FX}_{\mathrm{A}}$ as the temperature increases. It was also found that the lifetime of $\mathrm{FX}_{\mathrm{B}}$ is much shorter than that of $\mathrm{FX}_{\mathrm{A}}$ according to the timeresolved PL results. These results show that the light-hole free excitons in $\mathrm{GaN}$ can experience a stronger acousticphonon scattering and have a shorter lifetime than the heavyhole free excitons.

The work described in this paper was supported by the Joint Research Fund for Overseas Chinese, Hong Kong and Macau Scientists of NSFC (Grant No. 61028012), and partially by a grant from the University Grants Committee of the Hong Kong Special Administrative Region, China (Project No. AoE/P-03/08).

${ }^{1}$ M. Godlewski and E. M. Goldy's, in III-Nitride Semiconductors: Optical Properties II, edited by M. O. Manasreh and H. X. Jiang (Taylor \& Francis, New York, 2002), Chap. 7.

${ }^{2}$ A. Kasi Viswanath, J. I. Lee, S. Yu, D. Kim, Y. Choi, and C.-h. Hong, J. Appl. Phys. 84, 3848 (1998).

${ }^{3}$ W. Shan, T. Schmidt, X. H. Yang, J. J. Song, and B. Goldenberg, J. Appl. Phys. 79, 3691 (1996).

${ }^{4}$ G. D. Chen, M. Smith, J. Y. Lin, H. X. Jiang, S.-H. Wei, M. A. Khan, and C. J. Sun, Appl. Phys. Lett. 68, 2784 (1996).

${ }^{5}$ Y. J. Wang, R. X. Wang, G. Q. Li, and S. J. Xu, J. Appl. Phys. 106, 013514 (2009).

${ }^{6}$ J. Lagois, Phys. Rev. B 16, 1699 (1977).

${ }^{7}$ W. C. Tait and R. L. Weiher, Phys. Rev. 166, 769 (1968).

${ }^{8}$ S. Rudin, T. L. Reinecke, and B. Segall, Phys. Rev. B 42, 11218 (1990).

${ }^{9}$ S. J. Xu, L. X. Zheng, S. H. Cheung, M. H. Xie, S. Y. Tong, and H. Yang, Appl. Phys. Lett. 81, 4389 (2002).

${ }^{10}$ Y. Toyozawa, J. Phys. Chem. Solids 8, 289 (1959). 